\title{
Modulation of Crack Generation inside a LiF Single Crystal by Interference of Laser Induced Stress Waves
}

\author{
M. Sakakura ${ }^{1}$, Y. Ishiguro ${ }^{2}$, N. Fukuda ${ }^{1}$, Y. Shimotsuma $^{2}$, K. Miura $^{2}$ \\ ${ }^{* 1}$ Hitachi Zosen Collaborative Research Division, Office of Society-Academia Collaboration for In- \\ novation, Kyoto University, Kyoto, 615-8245, Japan \\ E-mail:msakakura@saci.kyoto-u.ac.jp \\ *2 Department of Material Chemistry, Graduate School of Engineering, Kyoto University, Kyoto, \\ 615-8510, Japan
}

\begin{abstract}
When a femtosecond laser pulse is focused inside a LiF single crystal, four cracks are generated in the $<100>$ directions from the photoexcited region. The time-resolved observation have elucidated that the tensile stress in the laser induced stress wave induces the generation and propagation of the cracks. In this study, to investigate the possibility of control of laser induced cracks inside single crystals, the interference of stress waves were generated by simultaneous fs laser irradiation at multiple spots inside a LiF crystal, and the effects to the crack formation were investigated. We found that the crack lengths depended on the photoexcited spots' distribution. The dependence was explained by different transient density distributions by different interference of stress waves. The simulated density distribution indicated that a compressive stress at a crack tip could prevent the crack from propagating further and an expansive stress along a crack could facilitate the crack propagation.
\end{abstract}

DOI: $10.2961 /$ jlmn.2014.01.0004

Keywords: Femtosecond laser processing, bulk machining, dynamics, crack, stress wave, single crystal, holographic laser processing

\section{Introduction}

Laser induced-modification depends on various properties of the target materials [1-4]. In particular, the atomic arrangement of the target (crystal or amorphous, crystal system and so on) is one of the important factors to determine the shape of the modification, because it determines the anisotropy of elastic constants and mechanical strength, which are responsible for the directions of fractures [5]. For example, after photoexcitation inside a LiF single crystal by a focused femtosecond laser pulse, a void-like structure appears at the photoexcited region and cracks are formed in the four $<100>$ directions from the photoexcited region as shown in Figure $1[3,6,7]$. In addition, the regions with birefringence, which are originated from dislocations, are observed along the $<110>$ lines from the photoexcited region in the crossed-Nicols image. In our previous study, we observed dynamics of fs a laser induced modification inside a LiF single crystal, and found that the transverse stress waves are responsible to the propagation of laser induced-cracks [7, 8]. This observation suggests that the crack formation could be inhibited or stimulated by modifying laser-induced stress wave, because the stress distribution can be modified by interference of a number of stress waves.

A number of stress waves can be generated easily by holographic laser machining system, in which a spatially phase modulated laser beam is focused in a target to generate multiple focal spots $[9,10]$. Therefore, the effect of the interference of laser induced-stress waves on the crack formations inside a single crystal can be investigated by the holographic fs laser machining system. In this study, we chose a LiF single crystal as a model material for investigating crack formation mechanism. In this paper, we first show that the crack formation is accompanied with a stress wave generation inside a LiF crystal after fs laser irradiation by a pump-probe optical microscope. Next, we show the crack formation inside a LiF crystal after irradiation with fs laser pulses at multiple spots and the difference in crack lengths by focal spots' distribution. Finally, based on the simulation of stress distributions, the origin of difference in crack lengths will be discussed. (a)

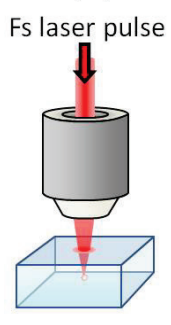

(b)

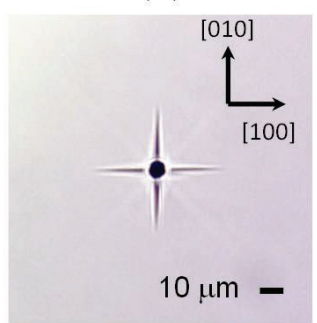

(c)

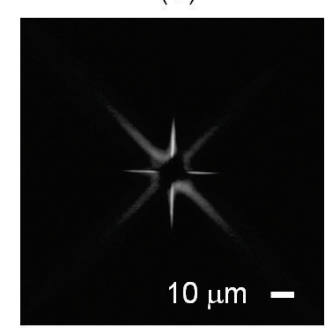

Fig. 1 (a) Schematic illustration of fs laser irradiation inside a LiF single crystal. (b) Transmission optical microscope images of modification inside a LiF single crystal after irradiation with focused fs laser pulse. The central black point is photoexcited region and four line-shaped structures in the $<100>$ directions are cracks. (c) The crossed-Nicols image of (b). The polarization orientation was parallel to the [100]. The bright regions indicate birefringence in the regions. 


\section{Method}

\subsection{Time-resolved observation of strain distribution}

The experimental setup of time-resolved observation of stress distribution is shown in our previous publication [8]. Briefly, an amplified fundamental pulse of a mode-locked Ti:sapphire laser from Mira-Legend (Coherent Inc.) was used as a pump pulse. The central wavelength of the laser pulse was $800 \mathrm{~nm}$ and the pulse duration was about $120 \mathrm{fs}$. The laser pulse was divided into two by a beam splitter, and one was used as a pump pulse and another was passed through a $\mathrm{BBO}\left(\beta-\mathrm{BaB}_{2} \mathrm{O}_{4}\right)$ crystal to generate the second harmonic pulse, which was used as a probe pulse. The pump pulse was focused inside a LiF single crystal with a $50 \times$ objective lens (Nikon, LU Plan, NA=0.80). The probe pulse was delayed by an optical delay stage and passed through a polarizer and a quarter-wave plate (QWP1) to be a circularly polarized light. The circularly polarized probe pulse was transmitted through the photoexcited region in the sample, and the transmitted light was reflected by a dichroic mirror and imaged on a charge coupled device (CCD) camera (HAMAMATSU; C10054-03) by the objective lens and a focusing lens (the focus length was 400 $\mathrm{mm}$.).

The sample was a LiF single crystal, in which the (001) surface had been cleaved and optically polished. The incident direction of the pump pulse was normal to the (001) surface, and the pump pulse was focused at about $150 \mu \mathrm{m}$ depth from the surface.

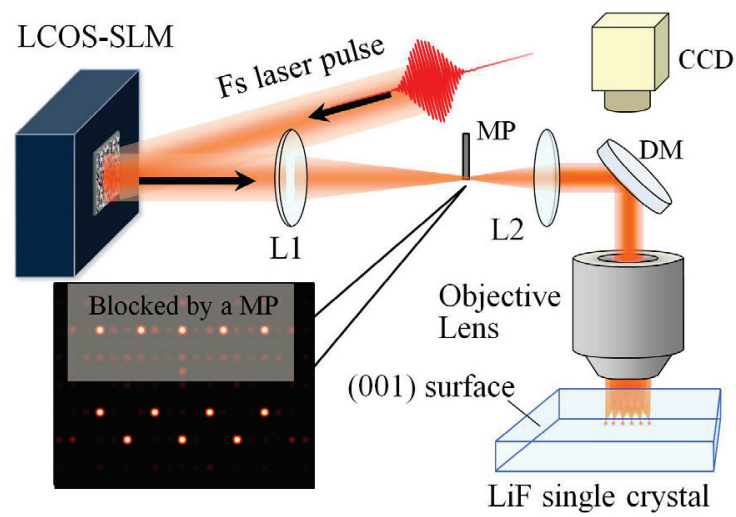

Fig. 2 Schematic illustration of optical setup of a holographic laser processing system. L1, L2 are lenses. The focal lengths are $500 \mathrm{~mm}$ and $150 \mathrm{~mm}$, respectively. MP is a metal plate, which was used to block a 0th order diffraction and unnecessary light spots. DM is a dichroic mirror. CCD is a charge coupled device camera.

\subsection{Holographic laser processing system}

A holographic laser processing system was used to induce photoexcitation simultaneously at multiple spots $[9$, 10]. The optical setup is shown in Fig. 2. Amplified Ti: $\mathrm{Al}_{2} \mathrm{O}_{3}$ fs laser pulses (Mira-Legend, Coherent Inc.) with a pulse duration of about 120 fs (FWHM), central wavelength of $800 \mathrm{~nm}$ and repetition rate of $1 \mathrm{kHz}$ were reflected on a LCOS-SLM (LCOS-SLM, X10468-02, Hamamatsu Photonics K.K.) [11]. The SLM modulated the spatial phase distributions of the laser pulses. The laser pulses after reflecting on the SLM passed through a telescope (the magnification is $\mathrm{M}=0.3$ ), and were focused inside a $\mathrm{LiF}$ single crystal with an objective lens $(f=4 \mathrm{~mm}, \mathrm{NA}=0.85$; LU-Plan, Nikon). The photoexcited regions were at about $150 \mu \mathrm{m}$ from the surface of a sample. A metal plate inside the telescope was used to eliminate a $0^{\text {th }}$ order diffraction spot and unnecessary light spots. Multiple focal spots were formed inside a sample, depending on the spatial phase modulation by the SLM. The phase distribution was calculated to generate a desired light spots' distribution by the optimal rotation angle (ORA) method [12], and sent to the SLM from a personal computer. The pulse energy of the laser beam was controlled with a neutral density (ND) filter, and the exposure time was controlled with a mechanical shutter.

\subsection{Simulation of laser induced stress wave}

The photoexcitation inside a crystal by a focused fs laser pulse induce rapid temperature elevation in the photoexcited region and the temperature elevation generates thermal stress. Subsequently, the thermal stress generates a stress wave as a result of stress relaxation process. Here, we simulated the stress wave generation based on the elastic dynamics $[1,7]$. We assumed that the stress came from the thermal stress in the multiple photoexcited points. For simplicity, the temperature change was expressed by

$$
\Delta T(t, \mathbf{r})=\sum_{l=1}^{N} \Delta T \exp \left\{-\left(\left|\mathbf{r}-\mathbf{r}_{l}^{\text {center }}\right| / w_{t h}\right)^{2}\right\} *\left\{1-\exp \left(-t / \tau_{t h}\right)\right\}
$$

(1),

where $t$ is the time after the temperature increase due to photoexcitation, $\boldsymbol{r}\{=(x, y, z)\}$ is the position, $l$ and $N$ are the index and number of the photoexcited points, respectively, $\Delta T$ is the temperature increase at the center, $\boldsymbol{r}_{l}^{\text {center }}$ is the central position of the $l$ th photoexcited point, $w_{t h}$ is the radius of the temperature distribution, and $\tau_{t h}$ is the rise time of the temperature change. The thermal diffusion during stress relaxation is negligible, because the time scale of the thermal diffusion is slower $(\sim \mu \mathrm{s})$ than the stress relaxation time $(\sim \mathrm{ns})$ [1]. We assumed $\tau_{\mathrm{th}} \sim 1 \mathrm{ps}$, because the generation of thermal stress have not been observed after several picoseconds in our previous study [7]. In fact, exact value of $\tau_{t h}$ is not so important for this simulation, because the relaxation of thermal stress occurs in several tens picoseconds, which is much longer than $\tau_{t h}$. We used $w_{t h}=2 \mu \mathrm{m}$, which was estimated by the void size at the photoexcited region inside a $\mathrm{LiF}$ single crystal.

Based on the elastic mechanical theory [1, 13], the response to the temperature change of the anisotropic material can be calculated by the following wave equation:

$$
\rho \frac{\partial^{2} \mathbf{u}(t, \mathbf{r})}{\partial t^{2}}=\nabla \cdot \mathbf{P}(t, \mathbf{r})-B \beta \nabla\{\Delta T(t, \mathbf{r})\}(2),
$$

where $\rho$ is the density, $\mathbf{u}(t, \boldsymbol{r})$ is the displacement vector, $\nabla=(\partial / \partial x, \partial / \partial y, \partial / \partial z), \mathbf{P}(t, \boldsymbol{r})$ is the stress tensor, B is the bulk modulus, and $\beta$ is the thermal expansion coefficient. The stress tensor is expressed by the elastic tensor of the crystal $(\boldsymbol{C})$ and the strain tensor $\{\boldsymbol{E}(t, \boldsymbol{r})\}$ as follows:

$$
\mathbf{P}(t, \mathbf{r})=\mathbf{C E}(t, \mathbf{r})(3) \text {. }
$$

The strain tensor, $\boldsymbol{E}(t, \boldsymbol{r})$, is the derivative of $\mathbf{u}(\mathrm{t}, \mathbf{r})$ with respect to $\mathbf{r}$. The parameters for the simulation are listed in Table 1 [14]. 
Table 1 Density $(\rho)$, thermal expansion coefficient $(\beta)$, and elastic constants $\left(\mathrm{C}_{11}, \mathrm{C}_{23}, \mathrm{C}_{44}\right)$ of a $\mathrm{LiF}$ single crystal

\begin{tabular}{cc}
\hline$\rho\left(\mathrm{gcm}^{-3}\right)$ & 2.64 \\
$\beta\left(\mathrm{K}^{-1}\right)$ & $3.7 \times 10^{-5}$ \\
$\mathrm{C}_{11}(\mathrm{GPa})$ & 110.7 \\
$\mathrm{C}_{23}(\mathrm{GPa})$ & 62.8 \\
$\mathrm{C}_{44}(\mathrm{GPa})$ & 45.7 \\
\hline
\end{tabular}

\section{Results and discussions}

\subsection{Time-resolved observation of crack formation}

Figure 3 shows time-resolved optical microscope images at various time delays after fs laser irradiation inside a LiF single crystal. The pulse energy was about $2 \mu \mathrm{J} /$ pulse. After the photoexcitation, a rounded-square stress wave propagated away from the photoexcited region at the center. The rounded-square shape of the stress wave is attributed to the anisotropy of the elastic tensor of a LiF crystal. The anisotropy of the elastic tensor results in directiondependent sound velocity; that in the $<110>$ direction is about 1.1 times larger than that in the $<100>$ [15]. The image at 1000 ps shows crack formations in the $<100>$ directions clearly. The cracks were elongated gradually and the elongation finished between 4000 ps to 5000 ps. This observation showed clearly that the crack formation was accompanied with the stress wave propagation. Faster propagation of a stress wave than elongation of cracks means that the origin of the crack elongation should be the stress wave. Therefore, the time-resolved observation suggests the possibility of control of the crack formation and elongation by interference of stress waves.

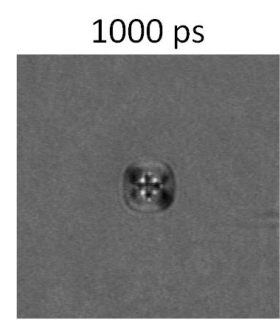

3000 ps

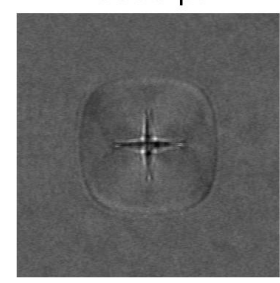

4000 ps
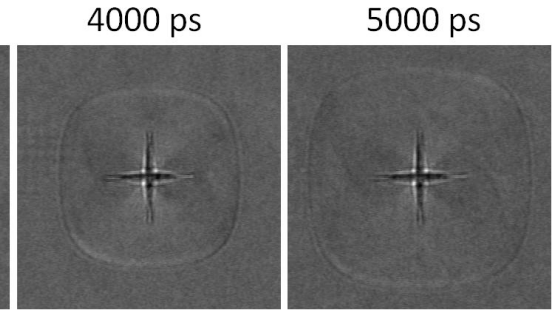

Fig. 3 Time-resolved transmission microscope images at various time delays after fs laser irradiation inside a $\mathrm{LiF}$ single crystal. The probe beam was circularly polarized and the polarization was analyzed by a quarter wave plate and an analyzer placed before a CCD camera.

\subsection{Crack formation by multiple spots' irradiation}

The distribution of focused light spots is shown in Fig. 4. Seven light spots were generated at the corners on a zig-zag line. The length of the line segment and the corner angle in the zig-zag were denoted by " $d$ " and " $\theta$ ', respectively. The photoexcited points were numbered as shown in Fig. 4.

We investigated the dependence of the crack length on $\theta$ at fixed $d(d \sim 34 \mu \mathrm{m})$. Figures 5 show the optical microscope images of cracks by focusing seven spots of fs laser pulses simultaneously inside a LiF single crystal. The images show that the lengths of cracks depended on $\theta$, position, and direction. For example, at $\theta=45^{\circ}$ and $90^{\circ}$, the cracks in the lower and upper directions were almost the same in length. On the other hand, while the cracks between the upper and lower photoexcited points' arrays were too short to be observed at $\theta=75^{\circ}$, the cracks in the same region were longer than that those in other regions at $\theta$ $>105^{\circ}$. In particular, the cracks in the lower direction from the spots "\#5", "\#6" and "\#7" and those in the upper direction from the spots " $\# 2$ " and " $\# 3$ " at $105^{\circ}$ were about two times longer than other cracks.

This remarkable dependence of the crack lengths on the spots' distribution suggests that different transient stress distributions should have affected the crack propagation. To confirm the difference in transient stress distributions, we calculated the transient density distributions of $\theta=75^{\circ}$ and $105^{\circ}$. Figures 6 show the simulated the transient stress distributions at the time when collision of stress waves modulated density distribution around the region of crack generation. At $\theta=75^{\circ}$, two stress waves began to collide around 2200 ps after the photoexcitation and the compressive stress appeared around the crack tip at 2500 ps [Fig. 6(a)]. On the other hand, in the simulation at $\theta=105^{\circ}$, compressive stress did not appeared at the crack tips at 2500 ps after laser irradiation [Fig. 6 (b), 2500 ps]. Instead, at $3500 \mathrm{ps}$, the tensile stress appeared along the region where a crack is generated [Fig. 6 (b)]. This tensile stress did not appeared at 3500 ps at $\theta=75^{\circ}$ because of different distribution of photoexcited points, as shown in Fig. 6(a).

In the experiment, it seemed that the cracks between the upper and lower photoexcited points' arrays had disappeared at $\theta=75^{\circ}$ [Fig. 5]. The simulated density distribution showed that the compressive stresses appeared at the tips of disappeared cracks at $2500 \mathrm{ps}$ after laser irradiation [Fig. 6 (a), 2500 ps]. This suggests that the compressive stress had shut the crack tip and prevented the crack from propagating further. On the other hand, at $\theta=105^{\circ}$, the cracks in the same regions were longer than those in other regions [Fig. 5]. The tensile stress in the simulated density distribution at 3500 ps [Fig. 6(b)] suggests that the tensile stresses had facilitated further propagation of the crack.
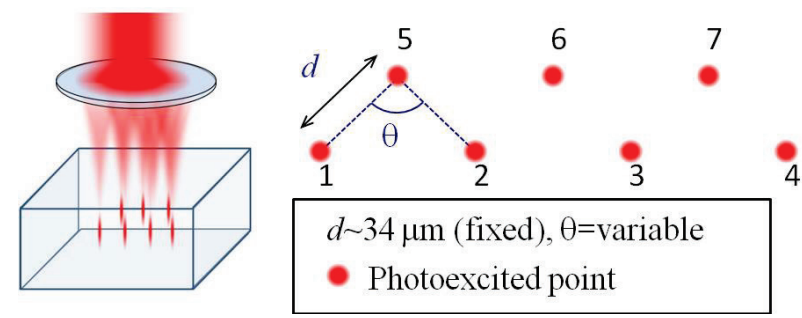

Fig. 4 Distribution of photoexcited points inside a sample. 


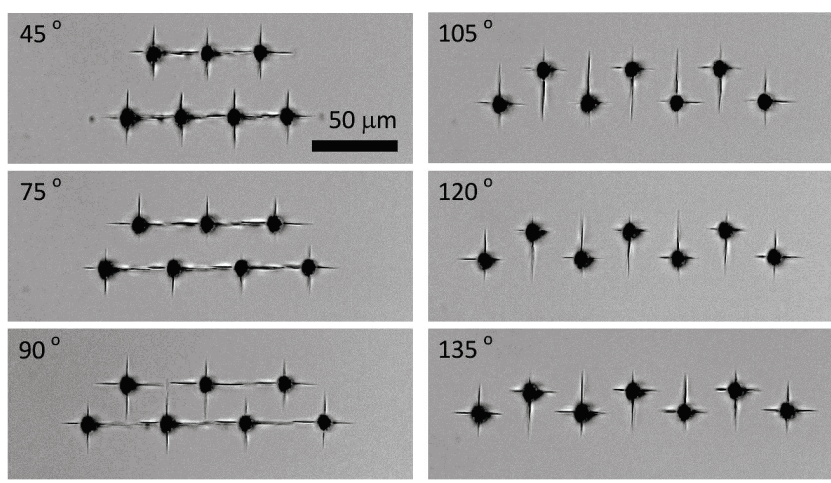

Fig. 5 Optical microscope images of cracks inside a LiF single crystal by focusing fs laser pulses at seven spots simultaneously. The seven spots are located at the corner of zig-zag lines of difference corner angle $(\theta)$. (a) $\theta=75^{\circ}$

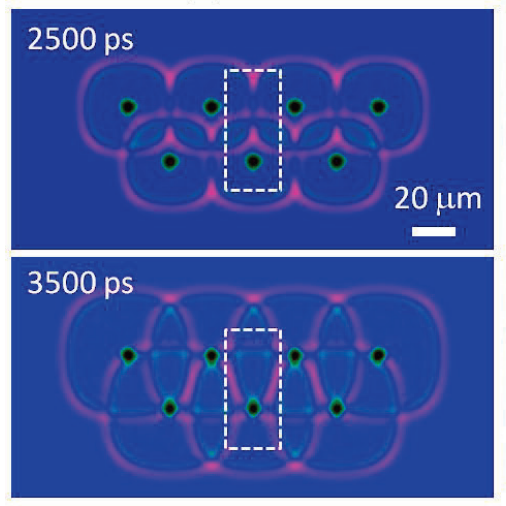

(b) $\theta=105^{\circ}$
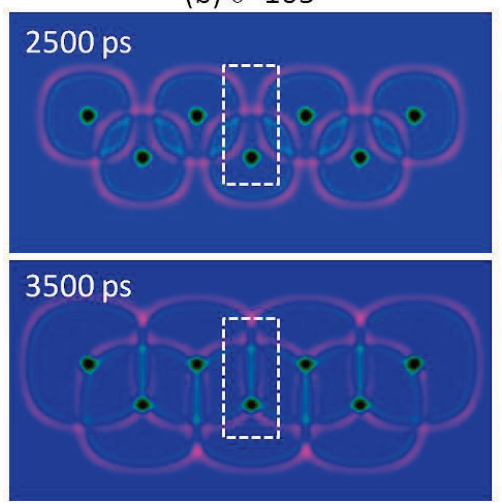

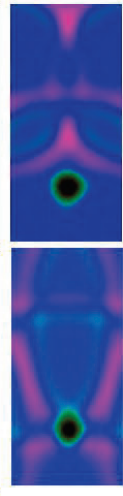

Density change (\%)

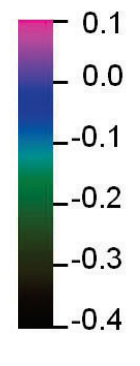

Fig. 6 Simulated transient density distributions after simultaneous photoexcitation at seven spots inside a LiF single crystal. The density distributions in the regions enclosed by white broken lines are magnified in the right figures.

\section{Conclusion}

We showed that the crack formation depended on the distribution of photoexcited points when these points were photoexcited simultaneously. The comparison between the crack length and the simulated transient density distribution suggests that the interference of stress waves could affect the crack propagation; the compression of material at the crack tip could prevent the crack from propagating and the expansion along the region of crack formation could facilitate the crack propagation. The results suggest that it is possible to control the crack formation by choosing the optimal distribution of the photoexcited points by a holographic laser processing.

\section{Acknowledgments}

This research was supported by Japan Society for the Promotion of Science (JSPS) Grant-in-Aid for Young Scientists (B), No. 22750187 and that for Scientific Research (A), No. 23246121.

\section{References}

[1] G. Paltauf and P. E. Dyer: Chem. Rev. 103, (2003) 487. (Journals)

[2] Z. Y. Wang, M. P. Harmer and Y.T. Chou: Materials Lett. 7 (1988) 224. (Journals)

[3] S. Kanehira, K. Miura, K. Fujita, K. Hirao, J. Si, N. Shibata and Y. Ikuhara: Appl. Phys. Lett. 90 (2007) 163110. (Journals)

[4] S. Juodkazis, K. Nishimura, S. Tanaka, H. Misawa, E. G. Gamaly, B. Luther-Davies, L. Hallo, P. Nicolai and V. T. Tikhonchuk: Phys. Rev. Lett. 96 (2006) 166101. (Journals) [5] W. D. Kingery, H. K. Bowen and D. R. Uhlmann: "Introduction to ceramics" (John Wiley \& Sons, Inc. 1976) Chaps. 4 and 14. (Books)

[6] B. Lawn: "Fracture of Brittle Solids" (Cambridge University Press, Cambridge, 1993) (Books)

[7] M. Sakakura, T. Tochio, M. Eida, Y. Shimotsuma, S. Kanehira, M. Nishi, K. Miura and K. Hirao: Opt. Express 19 (2011) 17780.

[8] T. Tochio, M. Sakakura, Y. Shimotsuma, M. Nishi, K. Hirao and K. Miura: Jpn. J. Appl. Phys. 51 (2012) 126602. (Journals)

[9] Y. Hayasaki, T. Sugimoto, A. Takita and N. Nishida: Appl. Phys. Lett. 87 (2005) 031101. (Journals)

[10] M. Sakakura, T. Sawano, Y. Shimotsuma, K. Miura and K. Hirao: Opt. Express 18 (2010)12136. (Journals) [11] T. Inoue, H. Tanaka, N. Fukuchi, M. Takumi, N. Matsumoto, T. Hara, N. Yoshida, Y. Igasaki and Y. Kobayashi: Proc. SPIE, 6487 (2007) Y11. (Journals)

[12] J. Bengtsson, Appl. Opt. 33, 6879-6884 (1994).

[13] L. D. Landau and E. M. Lifshitz: "Theory of elasticity" (Pergamon, Oxford, 1986). (Books)

[14] C. V. Briscoe and C. F. Squire: Phys. Rev. 106 (1957) 1175. (Journals)

[15] M J P Musgrave: Rep. Prog. Phys. 22(1959) 74. (Journals)

(Received: July 22, 2013, Accepted: December 15, 2013) 